\title{
Readiness of Patients with Multiple Sclerosis (MS) to Use Mobile Health Technology
}

\author{
Reza Abbasi ${ }^{1}$; Leila Ahmadian ${ }^{2}$; Nazanin Jannati ${ }^{3}$; Mohsen Baloochi ${ }^{1}$; Misagh Zahiri Esfahani ${ }^{1{ }^{1 *}}$ \\ ${ }^{1}$ MSc Student of Health Information Technology, Student Research Committee, Kerman University of Medical Sciences, Kerman, Iran \\ ${ }^{2}$ Associate Professor, Medical Informatics, Research Center for Social Determinants of Health, Institute for Futures Studies in Health, Kerman University of Medical Sciences, Ker- \\ man, Iran \\ ${ }^{3}$ MSc Student of Medical Informatics, Student Research Committee, Kerman University of Medical Sciences, Kerman, Iran \\ ${ }^{*}$ Corresponding author: Misagh Zahiri Esfahani, MSc Student of Health Information Technology, Student Research Committee in Kerman University of Medical Sciences, Ker- \\ man, P. O. Box: 7616911320, Iran. Tel: +98-9901876836, Fax:+98-3431325415, E-mail: misaghzahiri@yahoo.com
}

Received: 11 Dec 2016

Accepted: 01 Jan 2017

Epub: 23 Feb 2017

Ppub: 15 Jan 2018

\section{Abstract}

Background: Multiple sclerosis (MS) is a progressive disease of the central nervous system that has negative impacts on the quality of the patient's life. This disease is one of the most chronic disabling neurological diseases in young adults. The progress of this disease is unique and unpredictable. Self-care is one of the processes, which is used to control and prevent the progression of the disease. Some technologies such as mobile health can be used in improving monitoring as well as self-care in MS patients. Success in using this technology requires acceptance and readiness of the patients.

Objectives: The aim of this study was to evaluate the readiness of MS patients using mobile health technology.

Methods: This is a cross-sectional descriptive study that was conducted in 2016. Participants were MS patients who were referring to the MS clinic in Kerman city. We used convenient sampling method to recruit patients referred to the MS clinic during the study period. Data collection tool for this study was a questionnaire, which its validity was confirmed by 3experts in the fields of medical informatics and health information technology; its reliability coefficient was calculated $73 \%$.

Results: In total, 47 patients participated in the study. More than 55\% of them had an academic degree. More than 57\% of the patients had not heard about mobile health technology yet. More than $98 \%$ of patients stated that if the technology is free for them they are interested in using it. In addition, the same percentage of patients were feeling comfortable in terms of controlling their health situation by physicians or nurses via mobile phone. Also, more than $93 \%$ of these patients believed that the use of this technology can participate in effective communication with their physician. About $96 \%$ of patients agreed that the use of this technology can help them recall their medical orders.

Conclusions: According to the results, patients were interested and have the adequate readiness to use mobile health technology. This technology can improve the self-care of MS patients through effective communication between patients and health care providers. Furthermore, this technology can help patients recall their physicians' advices and orders and therefore increase their adherence.

Keywords: Mobile Health; Multiple Sclerosis; MS Disease; Readiness; Health Information System
\end{abstract}

